\title{
El tratamiento con indacaterol-glicopirronio fue más efectivo que salmeterol-fluticasona en la prevención de las exacerbaciones de la EPOC
}

Treatment with indacaterol-glycopyrronium was more effective than salmeterol-fluticasone in the prevention of COPD exacerbations

Wedzicha J. y col . N Engl J Med 2016;374:2222-34.

\section{Objetivos}

Comparar la cesación tabáquica gradual (CTG) con la abrupta (Comparar el tratamiento combinado de indacaterol-glicopirronio con salmeterol-fluticasona en la reducción de las exacerbaciones de la enfermedad pulmonar obstructiva crónica (EPOC).

\section{Diseño, lugar y pacientes}

Ensayo clínico aleatorizado, doble ciego, doble simulación (cada rama contenía tratamiento activo y placebo) y de no inferioridad. Estudio multicéntrico, realizado en 356 centros de 43 países. Se incluyeron pacientes con EPOC $\geq 40$ años, sintomáticos (Puntaje $\geq 2$ en la escala de disnea modificada MMRC1) con obstrucción moderada, grave y muy grave (VEF1 de $25 \%$ a $60 \%$ del valor predicho) que presentaron al menos una exacerbación el año previo.

\section{Intervención}

Se aleatorizaron 1.680 pacientes a recibir indacaterol $110 \mathrm{mcg}$ / glicopirronio $50 \mathrm{mcg}$ una vez por día (y placebo indistinguible de la rama activa) y 1682 pacientes a salmeterol $50 \mathrm{mcg} /$ fluticasona $500 \mathrm{mcg}$ dos veces por día (y placebo indistinguible de la otra rama activa) durante 52 semanas. Es decir que ambas ramas recibían beta agonistas de acción prolongada (del inglés LABA) pero en una rama recibían el antimuscarínico de acción prolongada (del inglés LAMA) glicopirronio y en la otra el corticoide fluticasona. Un periodo de una semana de rastreo seguido de un periodo de pre-inclusión o "run-in" de 4 semanas de tratamiento con $18 \mathrm{mcg}$ de tiotropio una vez al día. Posteriormente el tiotropio se discontinuó y los pacientes fueron asignados al azar, en una proporción de 1:1.

\section{Medición de resultados principales}

El resultado primario fue la tasa anual de exacerbaciones y los secundarios fueron: tiempo a la primera exacerbación, tiempo a la exacerbación moderada o severa, tiempo a la exacerbación severa, tasa anual de exacerbaciones moderadas o severas y severas, puntuación del cuestionario respiratorio St. George (SGRQ), valor de VEF1 y eventos adversos entre otros. Para el resultado primario se llevó a cabo un análisis por intención de tratar modificado y por protocolo, en cambio los resultados secundarios fueron analizados sólo por intención de tratar modificado. La población de intención de tratar modificada incluyó a todos los pacientes aleatorizados que recibieron al menos una dosis de un fármaco durante el período de tratamiento y no tuvieron violaciones mayores del cumplimiento de las guías de Buenas Prácticas Clínicas antes de que se produjera el desenmascaramiento. El margen de no inferioridad consideraba una razón de tasas de 1,15 y el de superioridad de 1 .

\section{Resultados principales}

El indacaterol-glicopirronio mostró superioridad respecto al salmeterol-fluticasona en la reducción de la tasa anual de exacerbaciones (ver tabla 1 ).

En los resultados secundarios se demostró que el grupo de indacate-

\begin{tabular}{|c|c|c|c|c|c|c|c|}
\hline \multirow[b]{2}{*}{ Resultado } & \multirow[b]{2}{*}{ Análisis } & \multicolumn{2}{|c|}{ Indacaterol-glicopirronio } & \multicolumn{2}{|c|}{ Salmeterol-Fluticasona } & \multirow{2}{*}{$\begin{array}{c}\text { RRR } \\
\text { (IC95\%) }\end{array}$} & \multirow{2}{*}{ P Valor } \\
\hline & & (IC 95\%) & $\mathbf{N}$ & (IC 95\%) & $\mathbf{N}$ & & \\
\hline Exacerbaciones & Por protocolo & $3,59(3,28$ a 3,94$)$ & 1.675 & $4,03(3,68$ a 4,41$)$ & 1.679 & $11 \%$ (4 a $17 \%)$ & 0,003 \\
\hline anuales & Por intención de tratar modificada & $3,59(3,29$ a 3,92$)$ & 1.528 & $4,09(3,75$ a 4,46$)$ & 1.556 & $12 \%(6$ a $8 \%)$ & 0,001 \\
\hline
\end{tabular}

IC 95\%: intervalo de confianza del 95\%. RRR: Reducción de riesgo relativo.

rol-glicopirronio tuvo más tiempo hasta la primera exacerbación que el grupo de salmeterol-fluticasona, hazard ratio 0,84 (IC 95\% 0,78 a 0,91 ). La tasa anual de exacerbaciones moderadas/severas fue $17 \%$ menor en el grupo de indacaterol-glicopirronio que con salmeterolfluticasona, razón de tasas 0,83 (IC95\% 0,75 a 0,91). La incidencia de efectos adversos fue similar, aunque en el grupo de salmeterolfluticasona la incidencia de neumonía fue mayor ( 4,8 vs $3,2 \%$ )

\section{Conclusiones}

En pacientes con EPOC y exacerbaciones en el año previo, el tratamiento con indacaterol-glicopirronio fue más efectivo que salmeterol-fluticasona en prevenir las exacerbaciones y sin aumento de efectos adversos.

Fuentes de Financiamiento: Novartis

\section{Comentario}

Los objetivos del tratamiento farmacológico de la EPOC son reducir la disnea y el riesgo de exacerbaciones. La guía GOLD 2017 recomienda el uso de broncodilatadores según la disnea del paciente, la historia de exacerbaciones y el VEF ${ }^{1.2}$ Estudios previos han demostrado que la combinación de salmeterol-fluticasona y el tiotropio presentan un efecto similar en la reducción de las exacerbaciones pero con mayor riesgo de neumonía con los corticoides inhalados. ${ }^{3}$ Posteriormente los estudios LANTERN y FLAME han demostrado que el tratamiento con indacaterol-glicopirronio es superior al de salmeterol-fluticasona en la reducción de las exacerbaciones ${ }^{4,5}$ Además en el estudio TRILOGY la triple terapia (beclometasona-formoterol-glicopirronio) demostró ser más efectiva en la reducción de las exacerbaciones que el tratamiento con beclometasona-formoterol. ${ }^{6}$ La comparación de la triple terapia con la terapia dual LAMA-LABA aún no ha sido evaluada. Por lo tanto en los pacientes que presentan disnea, la primera elección es la monoterapia con LABA (indacaterol) o LAMA (bromuro de tiotropio o glicopirronio) con preferencia de este último en aquellos que presentan exacerbaciones. En aquellos que son muy sintomáticos y se exacerban frecuentemente se sugiere la terapia dual con LABA-LAMA sobre corticoides inhalados-LABA por mejor perfil de eficacia y efectos adversos. Al momento se desconoce si la triple terapia puede aportar beneficios a la terapia de LAMA-LABA.

Conclusiones del comentador

La terapia dual con indacaterol-glicopirronio es el tratamiento de elección para los pacientes con EPOC y exacerbaciones ya que han demostrado ser más efectiva que el salmeterol-fluticasona en disminuirlas y con un menor riesgo de neumonías.

Horacio Matías Castro [ Sección de Neumonología, Servicio de Clínica Médica del Hospital Italiano de Buenos Aires. matias.castro@ hospitalitaliano.org.ar ] Castro HM. El tratamiento con indacaterol-glicopirronio fue más efectivo que salmeterol-fluticasona en la prevención de las exacerbaciones de la EPOC. Evid Act Pract Ambul. 2017;20(2):41. Comentado de: Wedzicha JA, Banerji D, Chapman KR, y col. Indacaterol-Glycopyrronium versus Salmeterol-Fluticasone for COPD. N Engl J Med 2016; 374:2222-34. PMID: 27181606. 2. Global strategy for the diagnosis, management, and prevention of chronic obstructive pulmonary disease, 2017 Report. Global Initiative for Chronic Obstructive Lung (GOLD).2017. 